\title{
On Shape Properties of the Receiver Operating Characteristic Curve
}

\author{
Bhaskar Bhattacharya \\ Department of Mathematics, Southern Illinois University \\ Carbondale, IL 62901, USA \\ bhaskar@siu.edu \\ and \\ Gareth Hughes \\ Scotland's Rural College, The King's Buildings \\ West Mains Road, Edinburgh, EH9 3JG, UK
}

\begin{abstract}
We present formal definitions of two commonly observed asymmetries in a concave receiver operating characteristic curve. The main theorem of the paper proves that the KullbackLeibler divergences between the underlying signal and noise variables are ordered based on these asymmetries. This result is true for any continuous distributions of the signal and noise variables.
\end{abstract}

Key words and phrases: Asymmetry, Kullback-Leibler divergence, entropy, relative distributions, $R O C$. 
1. Introduction. For binary diagnostic decisions, the receiver operating characteristic $(R O C)$ curve is a method for assessing the performance of procedures to classify subjects (or a received piece of information) as signal or noise. Imagine a single threshold on the scale of an underlying indicator variable. Then the group of subjects with indicator scores above the threshold denotes a prediction of need for treatment and the group of subjects with indicator scores below the threshold denotes a prediction of no need for treatment. Signals that are correctly classified are 'true positives', and noises that are correctly classified are 'true negatives'. Then the true positive proportion (TPP) expresses the number of true positives as a proportion of the total number of signals and the true negative proportion (TNP) expresses the number of true negatives as a proportion of the total number of noises. The false positive proportion is FPP $=1-$ TNP. Pairs of TPP and FPP values are obtained by allowing the threshold indicator score to vary over the whole range of the indicator variable. An $R O C$ curve is a graphical plot of TPP against FPP [11].

In this paper, we consider two absolutely continuous random variables (rvs), $X$ for signal and $Y$ for noise, with common support. Let $X$ have cumulative distribution function (cdf) $F_{1}$ and probability density function (pdf) $f_{1}$, and, those for $Y$ are $F_{2}$ and $f_{2}$, respectively. For any cdf $F$, we define the inverse function $F^{-1}(r)=\sup \{x: F(x) \leq r\}, 0 \leq r \leq 1$. Assume $F_{1}, F_{2}$ are invertible. It is well known that $R O C(t)=S_{1}\left(S_{2}^{-1}(t)\right), 0 \leq t \leq 1$ [11], where $S_{i}(x)=1-F_{i}(x), i=$ 1,2 . Then $R O C(t)$ is invertible. We assume that both $R O C$ and $R O C^{-1}$ are differentiable, and $R O C(t)$ is concave in $t$.

Using $X$ and $Y$, we define two new rvs, known as the relative rvs. Consider the transformation $U=F_{2}(X)$, which takes values in $(0,1) ; U$ is the relative rv which denotes the relative distribution for comparing $X$ with reference to $Y$ [6]. It is well known that the cdf and the pdf of $U$ are

$$
G_{1}(u)=F_{1}\left(F_{2}^{-1}(u)\right), \quad g_{1}(u)=\frac{f_{1}\left(F_{2}^{-1}(u)\right)}{f_{2}\left(F_{2}^{-1}(u)\right)}, 0 \leq u \leq 1,
$$

respectively. If the distributions of $X$ and $Y$ are identical, then the cdf of $U$ is a $45^{\circ}$ line and the pdf of $U$ is the uniform $(0,1)$ distribution.

Similarly, suppose $V=F_{1}(Y)$ is the relative $\mathrm{rv}$ which denotes the relative distribution for comparing $Y$ with reference to $X$, then the cdf and the pdf of $V$ are $G_{2}(v)=F_{2} F_{1}^{-1}(v), \quad g_{2}(v)=$ 
$\frac{f_{2}\left(F_{1}^{-1}(v)\right)}{f_{1}\left(F_{1}^{-1}(v)\right)}, 0 \leq v \leq 1$, respectively. Here $G_{1}, G_{2}$ are invertible, as $F_{1}, F_{2}$ are so.

For continuous signal and noise populations with pdfs $f_{1}(x), f_{2}(x), x \in \mathcal{D} \subset \Re$, respectively, the Kullback-Leibler divergence (KLD) between $f_{1}(x), f_{2}(x)$ is defined as $I\left(f_{1} \mid f_{2}\right)=$ $\int f_{1}(x) \ln \left(\frac{f_{1}(x)}{f_{2}(x)}\right) d x$. Although $I\left(f_{1} \mid f_{2}\right)$ is not a metric, it is well known that $I\left(f_{1} \mid f_{2}\right) \geq 0$ and $I\left(f_{1} \mid f_{2}\right)=0$ if and only if $f_{1}(x)=f_{2}(x), \forall x \in \mathcal{D}$. Hence it is often interpreted as a measure of 'divergence' or 'distance' between $f_{1}$ and $f_{2}$. Here and in the sequel, we observe the conventions that $\ln 0=-\infty, \ln (a / 0)=+\infty(a>0), 0 \cdot( \pm \infty)=0$. Interchanging the roles of $f_{1}$ and $f_{2}$, one could define $I\left(f_{2} \mid f_{1}\right)$ in a similar manner. However, it is well known that, in general, $I\left(f_{1} \mid f_{2}\right) \neq I\left(f_{2} \mid f_{1}\right)$.

Entropy (or differential entropy) of an $\mathrm{rv} X$ with cdf $F(x)$ (absolutely continuous) and pdf $f(x)$ is defined (and expressed) as $H(X)=-\int \ln f(x) d F(x)=-\int f(x) \ln f(x) d x$. Entropy is a measure of disparity of a density function $(f(x))$ from the uniform distribution. It measures the uncertainty of $X$ in the sense of utility of using $f(x)$ in place of the ultimate uncertainty of the uniform distribution [4]. For discrete rvs, entropy is nonnegative. However for a continuous rv $X$, entropy takes values in $[-\infty, \infty]$.

2. Examples and motivation. To see how changes in the shape of an $R O C$ curve correspond to changes in KLD, consider Figure 1 and Table 1. In Figure 1, each of the three boxes (unit squares), has three $R O C$ curves drawn in it. The area inside the box is referred to as the $R O C$ space. Now imagine the line joining points $(0,1)$ and $(1,0)$ of the unit square, known as the negative diagonal. When the $R O C$ curve is symmetric about the negative diagonal, it is called a symmetric $R O C$, otherwise it is called an asymmetric ROC. Each box in Figure 1 (A, B or C) has one symmetric (solid line) and two asymmetric (dashed or dotted line) ROC curves in it. The underlying signal and noise distributions (normal, lomax or beta) giving rise to these $R O C$ curves are listed in Table 1.

As in Section 1, recall $f_{1}$ : signal, $f_{2}$ : noise. Also, Table 1 lists the KLDs $\left[I\left(f_{1} \mid f_{2}\right)\right.$ and $\left.I\left(f_{2} \mid f_{1}\right)\right]$ corresponding to the distributions $f_{1}, f_{2}$. In each case $(\mathrm{A}, \mathrm{B}$ or $\mathrm{C})$, we see from Table 1 that for symmetric $R O C$, the KLDs are equal (measured in a unit called 'nits').

To describe asymmetric ROC curves, we consider true positive or negative proportion (TPP 
or TNP) types; TPP-asymmetry happens when the $R O C$ is more inclined (or, 'skewed' e.g. in [16]) towards the TPP axis (y-axis in $R O C$ space) when compared with a symmetric $R O C$, and TNP-asymmetry happens when the $R O C$ is more inclined towards the top line in the $R O C$ space (whose equation is $y=1$ in Cartesian plane), often called the TNP axis, when compared with a symmetric $R O C$, as seen from Figure 1. For asymmetric $R O C \mathrm{~s}$, when we compare the KLDs from Table 1, we find: for TPP-asymmetry, $I\left(f_{1} \mid f_{2}\right)>I\left(f_{2} \mid f_{1}\right)$ holds, and for TNP-asymmetry, $I\left(f_{1} \mid f_{2}\right)<I\left(f_{2} \mid f_{1}\right)$ holds. These observations are validated in Theorem 5.

Researchers are motivated to study the symmetry and asymmetry properties of the $R O C$ curves because it leads to information about the underlying (unknown) signal and noise variables. An $R O C$ curve is called binormal if both signal and noise variables are normally distributed, say $N\left(\mu_{i}, \sigma_{i}^{2}\right), i=1,2$, respectively. Discussions in [5] considered a binormal symmetric $R O C$ when $\mu_{1}>\mu_{2}$ with $\sigma_{1}^{2}=\sigma_{2}^{2}$, and an asymmetric $R O C$, when $\mu_{1}>\mu_{2}$ with $\sigma_{1}^{2}>\sigma_{2}^{2}$. Also, [5] noted that an empirical assumption that the ratio of the increment in the mean to the increment in the standard deviation (which they denoted as ' $\Delta m / \Delta s$ ') being equal to 4 often provided a satisfactory description of the (TPP-)asymmetric ROC curve for the data they considered. Note that $\Delta m / \Delta s$ is not generally usable, since when $\Delta s=0$ (symmetric ROC), it is undefined. Discussions in [5] concentrated around TPP-asymmetry only, and did not consider the case of TNP-asymmetry. Thus the example discussed in Section 4 concerning TNP-asymmetry will not be explainable by $\Delta m / \Delta s$. However, TNP-asymmetry is easily detected by the measure proposed in this paper as shown.

Discussing binormal $R O C$ curves in a clinical context, [15], p.83, Fig.4.1 noted that nondiseased subjects often tend to have more homogeneous test results than do diseased ones, leading again to an $R O C$ curve which is TPP-asymmetric. In [15], Chapter 4, some ROC summary indices are presented, although the properties of symmetry and asymmetry are not connected with the signs of the measure as it is shown in this paper. In an algebraic context, [10] found an $R O C$ curve to be symmetric, the signal distribution must be an orientation-reversing involution of the noise distribution. Some properties of symmetric ROCs are discussed in [8].

It is shown in [9] that in the binormal (with $\mu_{1} \neq \mu_{2}$ but $\sigma_{1}^{2}=\sigma_{2}^{2}$ ) case, the $R O C$ curve is 

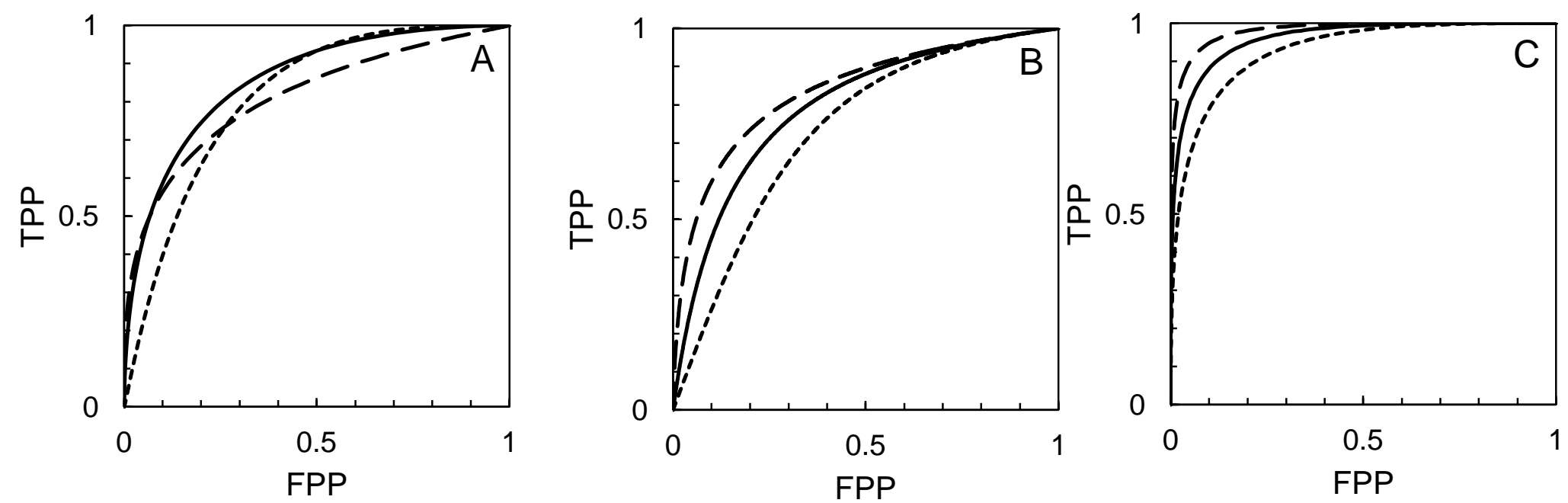

Fig. 1. ROC curves with varying parameters (Table 1). Solid line is symmetric ROC; dashed line is TPPasymmetric ROC; dotted line is TNP-asymmetric ROC. A. Binormal ROC curves. B. Lloyd's two-parameter lomax model ROC curves. C. Bi-beta ROC curves.
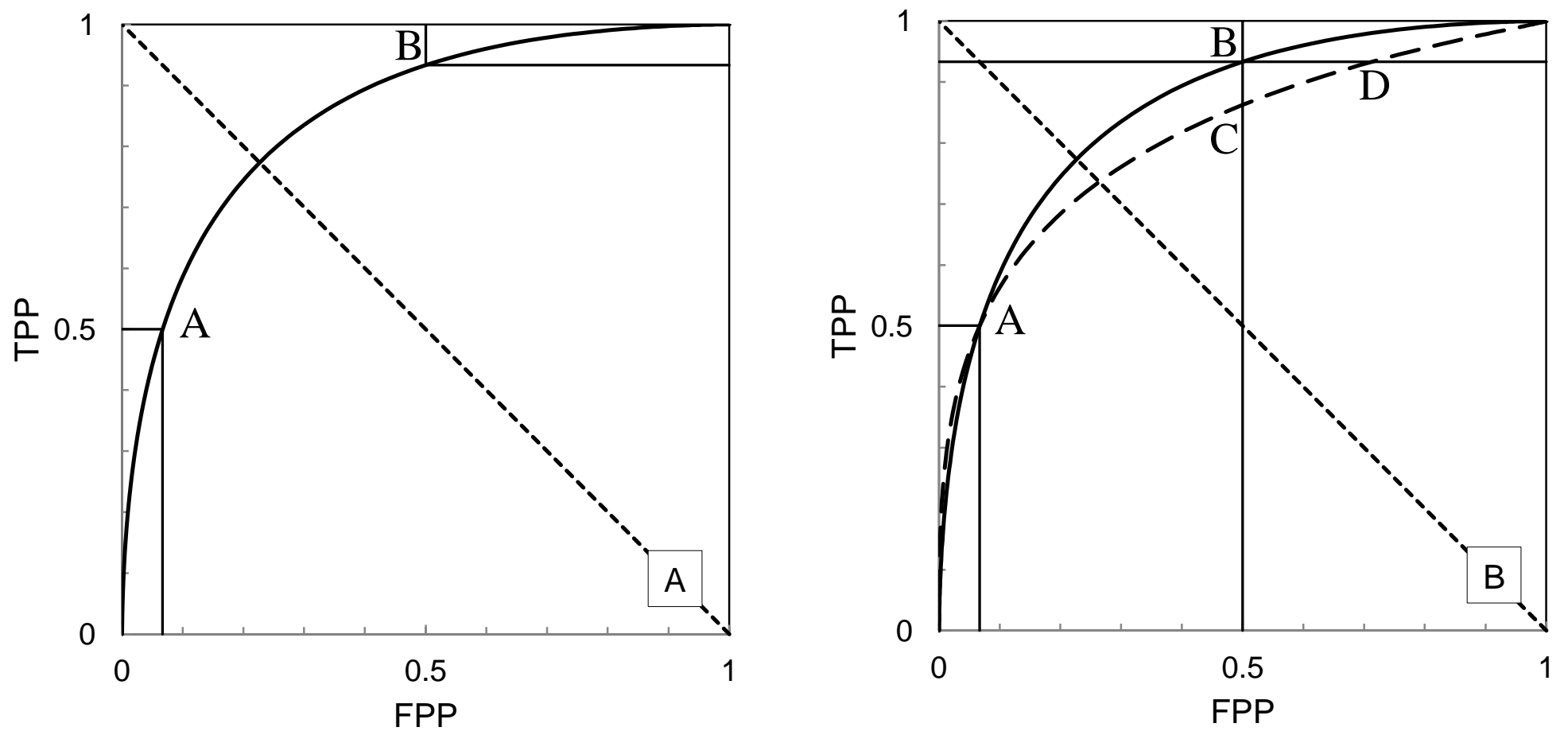

Fig. 2. A. Symmetry. $\mathrm{A}=(a, b), \mathrm{B}=\left(a_{1}, b_{1}\right)=(1-b, 1-a)$. B. TPP-asymmetry. $\mathrm{A}=(a, b), \mathrm{B}$ $=\left(a_{1}, b_{1}\right)=(1-b, 1-a), \mathrm{C}=\left(a_{2}, b_{2}\right)=\left(1-b, b_{2}<1-a\right), \mathrm{D}=\left(a_{3}, b_{3}\right)=\left(a_{3}>1-b, 1-a\right)$.

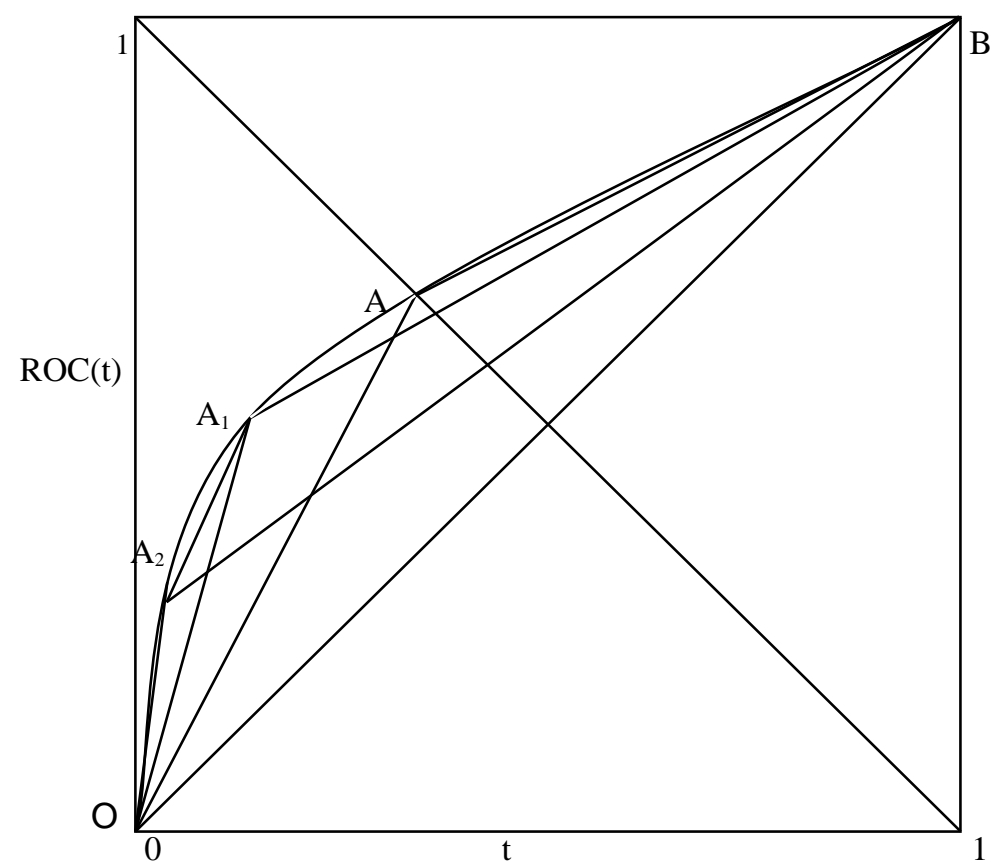

Figure 3. Discrete ROCs approximation of a TPP-asymmetric concave ROC, $A=(t, M t)$, $\mathrm{A}_{1}=\left(\mathrm{t}_{1}, \mathrm{M}_{1} \mathrm{t}_{1}\right), \mathrm{A}_{2}=\left(\mathrm{t}_{2}, \mathrm{M}_{2} \mathrm{t}_{2}\right)$ 
Table 1: Examples of distributions with $\mathrm{KLD}$ values and their $R O C$ types $\left(f_{1}\right.$ :signal, $f_{2}$ :noise)

\begin{tabular}{|c|c|c|c|c|}
\hline distribution & parameters & $I\left(f_{1} \mid f_{2}\right)$ & $I\left(f_{2} \mid f_{1}\right)$ & $R O C$ type \\
\hline \multirow{3}{*}{$\begin{array}{l}(\mathrm{A}) X \sim f_{1}=N\left(\mu_{1}, \sigma_{1}^{2}\right) \\
Y \sim f_{2}=N\left(\mu_{2}, \sigma_{2}^{2}\right) \\
\text { (Figure 1A) }\end{array}$} & $\begin{array}{l}\left(\mu_{1}, \sigma_{1}\right)=(1.5,1.0) \\
\left(\mu_{2}, \sigma_{2}\right)=(0.0,1.0)\end{array}$ & 1.125 nits $^{*}$ & 1.125 nits & symmetric \\
\hline & $\begin{array}{l}\left(\mu_{1}, \sigma_{1}\right)=(1.5,1.0) \\
\left(\mu_{2}, \sigma_{2}\right)=(0.0,1.375)\end{array}$ & 1.252 nits & 0.678 nits & TPP-asymmetric \\
\hline & $\begin{array}{l}\left(\mu_{1}, \sigma_{1}\right)=(5.5,1.0) \\
\left(\mu_{2}, \sigma_{2}\right)=(4.0,1.375)\end{array}$ & 0.678 nits & 1.252 nits & TNP-asymmetric \\
\hline \multirow{3}{*}{$\begin{array}{l}\text { (B) } f_{1}(x)=\frac{1}{\gamma b^{1 / \gamma}}(x+b)^{1+\frac{1}{\gamma}} \\
f_{2}(y)=\frac{1}{\gamma}(x+1)^{1+\frac{1}{\gamma}} \\
\text { lomax distribution } \\
\text { (Figure 1B) }\end{array}$} & $\Delta=\ln b=2, \gamma=1$ & 0.626 nits & 0.626 nits & symmetric \\
\hline & $\Delta=\ln b=2, \gamma=0.5$ & 1.056 nits & 0.823 nits & TPP-asymmetric \\
\hline & $\Delta=\ln b=2, \gamma=2.0$ & 0.347 nits & 0.417 nits & TNP-asymmetric \\
\hline \multirow{3}{*}{$\begin{array}{l}(\mathrm{C}) f_{1}(x)=\operatorname{Beta}\left(a_{1}, b_{1}\right) \\
f_{2}(x)=\operatorname{Beta}\left(a_{2}, b_{2}\right) \\
\text { (Figure } 1 \mathrm{C})\end{array}$} & $\begin{array}{l}\left(a_{1}, b_{1}\right)=(5,2), \\
\left(a_{2}, b_{2}\right)=(2,5)\end{array}$ & 3.251 nits & 3.251 nits & symmetric \\
\hline & $\begin{array}{l}\left(a_{1}, b_{1}\right)=(5.5,1.5) \\
\left(a_{2}, b_{2}\right)=(2,5)\end{array}$ & 4.853 nits & 4.457 nits & TPP-asymmetric \\
\hline & $\begin{array}{l}\left(a_{1}, b_{1}\right)=(4.5,2.5) \\
\left(a_{2}, b_{2}\right)=(2,5)\end{array}$ & 2.157 nits & 2.278 nits & TNP-asymmetric \\
\hline
\end{tabular}

*'nit' is a unit of measurement for information.

symmetric and two KLDs are equal. Also, [1] discusses general cases of symmetric $R O C$ curves for continuous data for which two KLDs are equal even though the signal and noise distributions themselves are not symmetric. The converse of this result is not true because equality of integrals does not imply equality of integrands. For some distributions other than those in Table 1, [7] discusses relations between $R O C$ and KLD. A close connection between $R O C$ and KLD is investigated by [3] in the context of likelihood ratios. In addition, there is an enormous literature on usage of $R O C$ curves together with KLDs across disciplines including medical, weather, linguistic, geology, chemistry and machine learning as can be found with internet search (e.g., [18], [19]). To learn more about $R O C$ curves, see [11], [15], [20]. 
3. Main results. Some interesting relationships between the cdfs $G_{1}, G_{2}, R O C$, KLDs are listed in the following Lemma. These relations will be useful later in the paper.

Lemma 1. Recall the definitions of $F_{i}, G_{i}, i=1,2$ from Section 1. Then the following relations hold: (i) $G_{1}(r)=G_{2}^{-1}(r), \forall r$, (ii) $R O C(r)=1-G_{1}(1-r), \forall r$, (iii) The relative rvs $U, V$ have only nonpositive entropies, and, (iv) $-H(U)=\int \log \left(\frac{\partial R O C(t)}{\partial t}\right) d t,-H(V)=\int \log \left(\frac{\partial R O C^{-1}(t)}{\partial t}\right) d t$. Proof: (i) This follows from the definitions of $G_{1}(r), G_{2}(r)$ given in Section 1, also see [6]. (ii) Let $F_{2}(x)=u$, then $x=F_{2}^{-1}(u)$. Since $S_{2}(x)=1-u$, we have $x=S_{2}^{-1}(1-u)$. Then $F_{2}^{-1}(u)=S_{2}^{-1}(1-u), \forall u$. Then $R O C(r)=S_{1} S_{2}^{-1}(r)=1-F_{1} F_{2}^{-1}(1-r)=1-G_{1}(1-r), \forall r$. (iii) In $I\left(f_{1} \mid f_{2}\right)=\int f_{1}(x) \ln \frac{f_{1}(x)}{f_{2}(x)} d x$, again let $F_{2}(x)=r$. Then $f_{2}\left(F_{2}^{-1}(r)\right) d x=d r$ holds, which together with (1) yields $0 \leq I\left(f_{1} \mid f_{2}\right)=\int g_{1}(r) \ln g_{1}(r) d r=-H(U)$. Similarly, $0 \leq I\left(f_{2} \mid f_{1}\right)=-H(V)$ holds (see [14]); then (iii) follows. Finally, (iv) follows using proof of (iii) and the fact that $I\left(f_{2} \mid f_{1}\right)=\int \log \left(\frac{\partial R O C(t)}{\partial t}\right) d t, I\left(f_{1} \mid f_{2}\right)=\int \log \left(\frac{\partial R O C^{-1}(t)}{\partial t}\right) d t$. (Result 1 in [1]).

From the definition of a symmetric ROC curve in Section 1, geometrically, it has the property that if $A=(a, b)$ (with $a+b \leq 1$ ) is a point on the curve, then $B=\left(a_{1}, b_{1}\right)=(1-b, 1-a)$ is also a point on the curve so that $A, B$ are reflections of each other about the negative diagonal. See Figure 2A. Then it follows (see [1]) that

$$
\left.\frac{\partial R O C(t)}{\partial t}\right|_{t=s}=\left.\frac{\partial R O C^{-1}(t)}{\partial t}\right|_{t=1-s}, \forall s \in(0,1) .
$$

Note that $\partial R O C(t) / \partial t$ is the likelihood ratio, see [5]. Recall the relative rv $U$ defined in Section 1. Now consider the rv $T=1-U$; let $T$ has cdf $G_{3}(t)$. One can verify easily that $G_{3}(t)=1-G_{1}(1-t)$, and the pdf is $g_{3}(t)=g_{1}(1-t), \forall t \in(0,1)$. Then the following holds.

Lemma 2. (a) When the $R O C$ curve is concave but asymmetric about the negative diagonal, then $G_{2}(t)$ and $G_{3}(t)$ are reflections of each other about the negative diagonal. (b) For a concave and symmetric $R O C, R O C(t)=1-G_{1}(1-t)=G_{2}(t)=G_{3}(t), \forall t$, holds. (c) For any shape $R O C$, there exists a $t^{*} \in(0,1)$ such that $t^{*}+R O C\left(t^{*}\right)=1$; then $G_{2}\left(t^{*}\right)=G_{3}\left(t^{*}\right)$ holds.

Proof. (a) Let $(a, b)$ be a point on $G_{3}$; we need to show that $(1-b, 1-a)$ is a point on $G_{2}$. Since $G_{3}(a)=1-G_{1}(1-a)=b$, we have $1-a=G_{1}^{-1}(1-b)=G_{2}(1-b)$. Hence the result. (b) This follows since $G_{3}(t)=R O C(t)$ by Lemma $1(\mathrm{ii})$, and then by (a), $G_{2}(t)=G_{3}(t), \forall t$. (c) If the $R O C(t)$ crosses the negative diagonal at $t=t^{*}$, then $t^{*}+R O C\left(t^{*}\right)=1$ holds. Since $G_{2}(t)$ 
and $G_{3}(t)$ are reflections of each other about the negative diagonal, so they must intersect on the negative diagonal, say at, $t=t^{*}$. Then $G_{2}\left(t^{*}\right)=G_{3}\left(t^{*}\right)$ holds.

We are interested in two types of asymmetries observed in $R O C$ curves, which are defined below. These definitions are obtained by considering a symmetric curve passing through a point on the asymmetric curve (point $A$ in Figure 2B). Each definition also refers to the point of intersection $\left(a^{*}, R O C\left(a^{*}\right)\right)$ of the asymmetric $R O C$ with the negative diagonal, see Lemma 2(c). In each case, two equivalent versions of the same definition are given depending on the locations of these two points.

Definition 1. A true positive proportion (TPP-) asymmetry happens when the ROC goes through $A=(a, b), a<a^{*}$ and $C=\left(a_{2}, b_{2}\right)$ where $a_{2}=1-b, b_{2}<1-a$, or $D=\left(a_{3}, b_{3}\right)$ where $a_{3}>1-b, b_{3}=1-a$. See Figure 2B. Also then, equivalently, the $R O C$ goes through $A=(c, d), c>a^{*}$ and $C=\left(c_{1}, d_{1}\right)$ where $c_{1}=1-d, d_{1}>1-c$, or $D=\left(c_{2}, d_{2}\right), c_{2}<1-d, d_{2}=$ $1-c$ (the second, equivalent to the first, description is not shown in figure $2 \mathrm{~B}$ to reduce clutter).

On the other hand, a true negative proportion (TNP-) asymmetry happens when the ROC goes through $A=(a, b), a<a^{*}$ and $D=\left(a_{3}, b_{3}\right)$ where $a_{3}=1-b, b_{3}>1-a$, or $a_{3}<1-b, b_{3}=$ $1-a$. Also then, equivalently, the $R O C$ goes through $A=(c, d), c>a^{*}$ and $C=\left(c_{2}, d_{2}\right)$ where $c_{2}=1-d, d_{2}<1-c$, or $c_{2}>1-d, d_{2}=1-c$. (not shown)

Recall from Lemma 1 (ii) that a $R O C$ curve can be expressed as $R O C(t)=1-G_{1}(1-t), 0 \leq$ $t \leq 1$. Also, from Lemma $2(\mathrm{~b})$, for a symmetric, concave $R O C$ curve, $1-G_{1}(1-a)=G_{2}(a), \forall a \in$ $(0,1)$ holds. The next lemma shows the relationship between $1-G_{1}(1-t)$ and $G_{2}(t)$ for TPPand TNP-asymmetric ROC curves.

Lemma 3. Assume the $R O C$ is concave. Let $G_{2}, G_{3}$ intersect at $a=a^{*}$ on the negative diagonal such that $G_{2}\left(a^{*}\right)=G_{3}\left(a^{*}\right)=1-G_{1}\left(1-a^{*}\right)$ holds. Then the following relations hold. (i) For a TPP-asymmetric $R O C$ curve, $1-G_{1}(1-a)>G_{2}(a)$ for $a<a^{*}, 1-G_{1}(1-a)<G_{2}(a)$ for $a>a^{*}, 0<a<1$. (ii) For a TNP-asymmetric ROC curve, $1-G_{1}(1-a)<G_{2}(a)$ for $a<a^{*}$, $1-G_{1}(1-a)>G_{2}(a)$ for $a>a^{*}, 0<a<1$.

Proof: (i) Consider $a<a^{*}$. Assume that $(a, b),\left(a_{2}, b_{2}\right)$ are on the TPP-asymmetric ROC. By definition $1, a_{2}=1-b, b_{2}<1-a$ hold. Then $b_{2}=R O C\left(a_{2}\right)=S_{1} S_{2}^{-1}\left(a_{2}\right)=S_{1} S_{2}^{-1}(1-b)<1-a$, 
or $1-F_{1} F_{2}^{-1}(b)<1-a$. From $(1)$, then $1-G_{1}(b)<1-a$, or, $G_{1}(b)>a$. Now use the fact that $b=R O C(a)=S_{1} S_{2}^{-1}(a)=1-F_{1} F_{2}^{-1}(1-a)=1-G_{1}(1-a)$, from which it follows that $G_{1}\left(1-G_{1}(1-a)\right)>a$, or $1-G_{1}(1-a)>G_{1}^{-1}(a)=G_{2}(a)$, from Lemma 1 (i). The case of $a>a^{*}$ follows similarly from the corresponding part of the definition of TPP-asymmetry. (ii) This is proved using similar technique as in (i).

In Theorem 5 below, we consider the case of a typical TPP-asymmetric $R O C$, as shown in Figure 3, which crosses the negative diagonal at the point $A$. If the slope of the line joining $(0,0)$ and $A$ is $M$, then $A=\left(\frac{1}{M+1}, \frac{M}{M+1}\right)$. The typical shape of the curve $R O C(t)$ for $t \in\left(0, \frac{1}{M+1}\right)$ is of interest. We approximate the curve shape of the $R O C$ in $\left(0, \frac{1}{M+1}\right)$ by a discrete $R O C$ (a piecewise linear function), formed by joining a series of $n$ points on the $\operatorname{ROC}\left(A_{1}, A_{2}, \ldots\right.$ etc.) as shown in Figure 3. As $n \rightarrow \infty$, these discrete ROCs (based on $n$ points) would converge to the continuous $R O C$ of interest in $\left(0, \frac{1}{M+1}\right)$ as shown by Theorem 5 below. First we prove Lemma 4, which will be needed in proving Theorem 5 .

Lemma 4. For $M_{2}>M_{1}>1,\left(1+M_{2}\right) \ln \frac{M_{2}}{M_{1}}-\frac{\left(1+M_{1}\right)\left(M_{2}-M_{1}\right)}{M_{1}}>0$.

Proof. Let $M_{2}=k M_{1}$, where $k>1$. Then $\left(1+M_{2}\right) \ln \frac{M_{2}}{M_{1}}-\frac{\left(1+M_{1}\right)\left(M_{2}-M_{1}\right)}{M_{1}}=\ln k+M_{1} k \ln k-$ $(k-1)-M_{1}(k-1)$. This quantity is 0 when $k=1$. To show the desired result when $k>1$, we differentiate the given quantity with respect to $k$, and we get $k^{-1}-1+M_{1} \ln k$. As $M_{1} \ln k>$ $\ln k>1-k^{-1}, \forall k>1$, the result follows.

The main result of this paper is presented in Theorem 5 below, where we assume that the following two properties hold:

(P1) for a TPP-asymmetric $R O C$, the derivative near 0 must be finite (for a TNP-asymmetric $R O C$, the derivative near 1 must be positive), and,

(P2) a TPP-asymmetric concave $R O C$, which can be well approximated by a straight line in the interval $\left(t^{*}, 1\right)$ (a TNP-asymmetric concave $R O C$, which can be well approximated by a straight line in the interval $\left.\left(0, t^{*}\right)\right)$, where $t^{*}+R O C\left(t^{*}\right)=1$.

We believe that these properties are not too restrictive. If a TPP-asymmetric $R O C$, say $R$, starts at $(0, a), a>0$, one might consider an approximating continuous TPP-asymmetric ROC by replacing the vertical line connecting $(0,0)$ to $(0, a)$ by a slightly slanted line with slope $1-\epsilon$, for 
$\epsilon>0$, say $R_{\epsilon}$, by choosing $\epsilon$ sufficiently small. Then Theorem 5 is applicable for the transformed ROC $R_{\epsilon}$. Also, (P2) is reasonable, since the main interest of a TPP-asymmetric $R O C$ curve is its shape in $\left(0, t^{*}\right)$ interval. This is true for the TPP-asymmetric ROCs in Figure 1.

Theorem 5. Under assumptions (P1) and (P2), for a TPP-asymmetric concave $R O C,-H(U) \geq$ $-H(V)$ holds. Similarly, for a TNP-asymmetric concave $R O C,-H(U) \leq-H(V)$ holds.

Proof. Consider the TPP-asymmetric ROC shown in Figure 3. We construct a sequence of discrete $R O C$ approximations to this TPP-asymmetric continuous $R O C$. Note that if $A=$ $(t, t M), 0<t<1, M>1$ is on $R O C(t)$, then $(t M, t)$ is on $R O C^{-1}(t)$.

Before we consider the TPP-asymmetric case, it is instructive to consider a one-point discrete symmetric $R O C$, say $R_{0}(t)=\mathrm{OAB}$, as shown in Figure 3. Since $A=(t, t M)$ is on the negative diagonal, we have $t+t M=1, M>1$, i.e., $t=\frac{1}{M+1}$. Then one can verify that $\frac{1-t M}{1-t}=\frac{1}{M}$ holds by substituting for $t$. The discrete $R O C R_{0}(t)$ has two line segments $\mathrm{OA}$ and $\mathrm{AB}$ with slopes $M, \frac{1-t M}{1-t}$, respectively. Also the inverse of $\mathrm{OAB}$ (or $R_{0}^{-1}(t)$ ) would have two line segments (not drawn) with slopes $\frac{1}{M}, \frac{1-t}{1-M t}$, respectively. Substituting the slopes of these line segments, $\int \log \left(\frac{\partial R_{0}(t)}{\partial t}\right) d t=t \ln M+(1-t) \ln \frac{1-t M}{1-t}=t \ln M-(1-t) \ln M$, and, $\int \log \left(\frac{\partial R_{0}^{-1}(t)}{\partial t}\right) d t=$ $M t \ln \frac{t}{M t}+(1-M t) \ln \frac{1-t}{1-M t}=-(1-t) \ln M+t \ln M$. Using Lemma 1(iv), $D_{0}=(-H(U))-$ $(-H(V))=\int \log \left(\frac{\partial R_{0}(t)}{\partial t}\right) d t-\int \log \left(\frac{\partial R_{0}^{-1}(t)}{\partial t}\right) d t=0$.

To approximate a TPP-asymmetric $R O C$ as in Figure 3, we first consider a one-point discrete TPP-asymmetric $R O C$, which we refer to as $R_{1}=O A_{1} B$ where $\left.A_{1}=\left(t_{1}, t_{1} M_{1}\right)\right), t_{1}+t_{1} M_{1}<$ 1, $M_{1}>1$. From the slopes of $O A_{1}$ and $A_{1} B$, and comparing those with the slopes of the inverse of $O A_{1} B$, we have, $\frac{1-M_{1} t_{1}}{1-t_{1}}>\frac{1}{M_{1}}$. As before, we have $D_{1}=D_{1}\left(t_{1}\right)=\int \log \left(\frac{\partial R_{1}(t)}{\partial t}\right) d t-$ $\int \log \left(\frac{\partial R_{1}^{-1}(t)}{\partial t}\right) d t=t_{1}\left(M_{1}+1\right) \ln M_{1}+\left(2-t_{1}-M_{1} t_{1}\right) \ln \frac{1-M_{1} t_{1}}{1-t_{1}}$. It is easy to verify as $t_{1} \rightarrow 0$ (or, $A_{1}$ approaches origin), and, as $t_{1} \rightarrow \frac{1}{M+1}$ (or $A_{1}$ approaches to $A$ on the negative diagonal), $D_{1} \rightarrow 0$. Since $\frac{\partial^{2} D_{1}}{\partial t_{1}^{2}}=\frac{-\left(M_{1}-1\right)^{3} t_{1}}{\left(1-t_{1}\right)\left(1-M_{1} t_{1}\right)}<0$ for $0<t_{1}, M_{1} t_{1}<1, M_{1}>1, D_{1}$ is concave in $t_{1}$. Then $D_{1}$ must be positive for $t_{1} \in\left(0, \frac{1}{M+1}\right)$, and negative for $t_{1} \in\left(\frac{1}{M+1}, 1\right)$.

Starting from the TPP-asymmetric $R_{1}$ in last step, we construct the second discrete TPPasymmetric $R O C$ (say, $R_{2}=O A_{2} A_{1} B$ ) by adding a second point $A_{2}=\left(t_{2}, M_{2} t_{2}\right)$ on $R O C$, with $t_{2}<t_{1}, M_{2}>M_{1}>1, t_{i}+M_{i} t_{i}<1, \forall i$. Thus $R_{2}$ is the piecewise linear function obtained by 
joining four points, namely, $(0,0), A_{2}, A_{1}$ and $(1,1)$. Thus, there are three line segments for each of $R_{2}$ and $R_{2}^{-1}$ (of which only the first two segments involve $t_{2}$ over intervals $\left(0, t_{2}\right),\left(t_{2}, t_{1}\right)$ ), and considering the slopes, we have

$$
\begin{aligned}
D_{2}= & D_{2}\left(t_{2}, t_{1}\right)=\int \log \left(\frac{\partial R_{2}(t)}{\partial t}\right) d t-\int \log \left(\frac{\partial R_{2}^{-1}(t)}{\partial t}\right) d t=t_{2}\left(M_{2}+1\right) \ln M_{2}- \\
& {\left[t_{1}\left(M_{1}+1\right)-t_{2}\left(M_{2}+1\right)\right] \ln \left(\frac{M_{1} t_{1}-M_{2} t_{2}}{t_{1}-t_{2}}\right)+\left(2-t_{1}-M_{1} t_{1}\right) \ln \left(\frac{1-M_{1} t_{1}}{1-t_{1}}\right) . }
\end{aligned}
$$

Note that only the first two terms involve $t_{2}$. Then

$$
\frac{d D_{2}}{d t_{2}}=\left(M_{2}+1\right) \ln \left(\frac{M_{2}\left(t_{1}-t_{2}\right)}{M_{1} t_{1}-M_{2} t_{2}}\right)+\frac{\left[t_{1}\left(M_{1}+1\right)-t_{2}\left(M_{2}+1\right)\right]\left(M_{1}-M_{2}\right) t_{1}}{\left(t_{1}-t_{2}\right)\left(M_{1} t_{1}-M_{2} t_{2}\right)} .
$$

For $t_{2}=0$ or $t_{1}$, the curve $R_{2}$ reduces to $R_{1}$, and we have $D_{2}=D_{1}>0$. As $t_{2} \rightarrow 0, \frac{d D_{2}}{d t_{2}}=$ $\left(1+M_{2}\right) \ln \frac{M_{2}}{M_{1}}-\frac{\left(1+M_{1}\right)\left(M_{2}-M_{1}\right)}{M_{1}}>0$ by Lemma 4 , since $M_{2}>M_{1}>1$. Thus $D_{2}$ is increasing in $t_{2}$ near 0 . Thus $D_{2}>0$ for $t_{2}$ near 0 .

Also, $\frac{d^{2} D_{2}}{d t_{2}^{2}}=\frac{\left(M_{2}-M_{1}\right)^{2}\left(M_{1} t_{1}-t_{1}+t_{2}-M_{2} t_{2}\right) t_{1}^{2}}{\left(M_{1} t_{1}-M_{2} t_{2}\right)^{2}\left(t_{1}-t_{2}\right)^{2}}$, which is $<0$ if $t_{2}<t_{20}=\frac{t_{1}\left(M_{1}-1\right)}{\left(M_{2}-1\right)}$, and $>0$ for $t_{2}>t_{20}$. Thus for fixed $t_{1}$, as a function of $t_{2}, D_{2}$ is concave for $t_{2}<t_{20}$, and convex for $t_{2}>t_{20}$. Thus we get $D_{2}>0$ for $0<t_{2}<t_{1}<\frac{1}{M+1}$. Note that $t_{20}<t_{1}$, and $t_{2}, t_{20}, t_{1}$ (or equivalently, $M_{2}, M_{1}$ ) could be arbitrarily close to each other.

Proceeding in this manner, assume that $D_{n-1}>0$ for some $n \geq 3$. To show $D_{n}>0, \forall n$, first define $R_{n}$ as the piecewise linear function obtained by joining points, $(0,0), A_{n}, \ldots, A_{2}, A_{1}$ and $(1,1)$, where $A_{n}=\left(t_{n}, M_{n} t_{n}\right)$. Then $D_{n}=\int \log \left(\frac{\partial R_{n}(t)}{\partial t}\right) d t-\int \log \left(\frac{\partial R_{n}^{-1}(t)}{\partial t}\right) d t$. To study $D_{n}$ as a function of $t_{n}$, it is enough to consider only the first two intervals $\left(0, t_{n}\right),\left(t_{n}, t_{n-1}\right)$ because the rest of the intervals do not involve $t_{n}$.

Considering the slopes of $R_{n}, R_{n}^{-1}$ over these intervals, we have $D_{n}=t_{n}\left(M_{n}+1\right) \ln M_{n}-$ $\left[t_{n-1}\left(M_{n-1}+1\right)-t_{n}\left(M_{n}+1\right)\right] \ln \left(\frac{M_{n-1} t_{n-1}-M_{n} t_{n}}{t_{n-1}-t_{n}}\right)+C_{n}$, where $C_{n}$ does not depend on $t_{n}$. Then

$$
\frac{d D_{n}}{d t_{n}}=\left(M_{n}+1\right) \ln \left(\frac{M_{n}\left(t_{n-1}-t_{n}\right)}{M_{n-1} t_{n-1}-M_{n} t_{n}}\right)+\frac{\left[t_{n-1}\left(M_{n-1}+1\right)-t_{n}\left(M_{n}+1\right)\right]\left(M_{n-1}-M_{n}\right) t_{n-1}}{\left(t_{n-1}-t_{n}\right)\left(M_{n-1} t_{n-1}-M_{n} t_{n}\right)} .
$$

For $t_{n}=0$ or $t_{n-1}$, the curve $R_{n}$ reduces to $R_{n-1}$, and we have $D_{n}=D_{n-1}>0$. As $t_{n} \rightarrow 0$, $\frac{d D_{n}}{d t_{n}}=\left(1+M_{n}\right) \ln \frac{M_{n}}{M_{n-1}}-\frac{\left(1+M_{n-1}\right)\left(M_{n}-M_{n-1}\right)}{M_{n-1}}>0$ by Lemma 4 , since $M_{n}>M_{n-1}>1$. Thus $D_{n}$ is increasing in $t_{n}$ near 0 . Thus $D_{n}>0$ for $t_{n}$ near 0 . 
Also, $\frac{d^{2} D_{n}}{d t_{n}^{2}}=\frac{\left(M_{n}-M_{n-1}\right)^{2}\left(M_{n-1} t_{n-1}-t_{n-1}+t_{n}-M_{n} t_{n}\right) t_{n-1}^{2}}{\left(M_{n-1} t_{n-1}-M_{n} t_{n}\right)^{n}\left(t_{n-1}-t_{n}\right)^{2}}$, which is $<0$ if $t_{n}<t_{n 0}=\frac{t_{n-1}\left(M_{n-1}-1\right)}{\left(M_{n}-1\right)}$, and $>0$ for $t_{n}>t_{n 0}$. Thus for fixed $t_{n-1}$, as a function of $t_{n}, D_{n}$ is concave for $t_{n}<t_{n 0}$, and convex for $t_{n}>t_{n 0}$. Thus we get $D_{n}>0$ for $0<t_{n}<t_{n-1}<\ldots<t_{1}<\frac{1}{M+1}$. Note that $t_{n 0}<t_{n-1}$, and $t_{n}, t_{n 0}, t_{n-1}$ (or equivalently, $M_{n}, M_{n-1}$ ) could be arbitrarily close to each other. Thus, $D_{n}>0, \forall n$.

By the above construction, the sequence of discrete $R O C \mathrm{~s}, R_{n}(t) \rightarrow R O C(t)$ for $t<\frac{1}{M+1}$ as $n \rightarrow \infty$, and by $(\mathrm{P} 2)$, for all $t$. Let $D_{n}=\int h_{n}(t) d t$, where $h_{n}(t)=\log \left(\frac{\partial R_{n}(t)}{\partial t}\right)-\log \left(\frac{\partial R_{n}^{-1}(t)}{\partial t}\right)$. Let $D=\int h(t) d t$ where $h(t)=\ln \frac{\partial R O C}{\partial t}(t)-\ln \frac{\partial R O C^{-1}}{\partial t}(t)$. Then, by continuity of 'ln', $h_{n}(t) \rightarrow h(t)$ as $n \rightarrow \infty$. Since the derivative of $R O C(t)$ near 0 must be finite by $(\mathrm{P} 1)$, so $h_{n}$ are uniformly bounded with uniformly bounded slopes. By Arzelà-Ascoli theorem, there is a subsequence $\left\{n_{j}\right\}$ such that $h_{n_{j}} \rightarrow h$ uniformly as $n_{j} \rightarrow \infty$. Then it follows that $D_{n_{j}}=\int h_{n_{j}}(t) d t \rightarrow \int h(t) d t=D$ as $n_{j} \rightarrow \infty$. Since $D_{n}>0, \forall n$, we get $D>0$. This proves the result.

4. Real-data example. Sclerotinia stem rot (caused by Sclerotinia sclerotiorum) is a soilborne fungal disease of oilseed rape and some other crops. Effective control is available by use of fungicides, but these must be applied before infection occurs so it is necessary to treat crops on the basis of calculation of disease risk rather than observation of disease symptoms. Over a 10-year period, [17] observed 805 individual oilseed rape crops, all untreated with fungicide. The crops were divided retrospectively into two groups on the basis of whether fungicide application would have been economically beneficial (131 crops, referred to as cases) or otherwise (674 crops, referred to as controls) (this is the gold-standard assessment). Then a risk score, calculated from observations of factors related to Sclerotinia stem rot disease risk was calculated for all crops, and frequency distributions of risk scores plotted separately for the case and control groups (Figure 2 in [17]). This analysis was the basis for calculation of an empirical ROC curve (Figure 1 in [17]). Visually, the empirical ROC curve appears to be slightly TNP-asymmetric. This is confirmed by analysis of the corresponding bi-Normal ROC curve (see supplement).

5. Final comments. In this paper we have shown that the relative entropies $I\left(f_{1} \mid f_{2}\right)$ and $I\left(f_{2} \mid f_{1}\right)$ can provide a useful two-figure summary of the shape properties of a $R O C$ curve. The most often used summary statistic for $R O C$ curves is the area under the curve (AUC). It is a 
one-figure summary and may not capture differences in shape (geometry) of $R O C \mathrm{~s}$ in the sense that $R O C$ curves that are symmetric, TPP-asymmetric and TNP-asymmetric may all have the same AUC. But, as we have shown, these ROCs may be distinguished on the basis of their KLDs. The symmetry/asymmetry properties discussed are valid for any continuous distributions (not only binormal $R O C \mathrm{~s}$ ) of the signal and noise distributions, as long as they produce a concave $R O C$.

Acknowledgments The first author would like to thank Professor Philip Feinsilver for several stimulating discussions and the National Science Foundation for partial support. Both authors would like to thank the coeditor and the referees for their suggestions which have helped to improve the presentation of the paper.

\section{References}

[1] Bhattacharya, B. and Hughes, G. (2011), Symmetry of receiver operating characteristic curves and Kullback-Leibler divergences between the signal and noise populations, Journal of Mathematical Psychology, 55, 365-367.

[2] Cover, T. M. and Thomas, J. (1991), Elements of Information Theory, Wiley, New York.

[3] Eguchi, S. and Copas, J. (2006), Interpreting Kullback-Leibler divergence with the NeymanPearson lemma, Journal of Multivariate Analysis, 90, no. 2, 317-336.

[4] Good, I. J. (1968), Utility of a distribution, Nature, 219, 51-61.

[5] Green, D. M. and Swets, J. A. (1966), Signal detection theory and psychophysics, Wiley, New York.

[6] Handcock, M. S., and Morris, M. (1998), Relative distribution methods, Sociological Methodology, 28, 53-97. 
[7] Hughes, G. and Bhattacharya, B. (2013), Symmetry Properties of Bi-Normal and Bi-Gamma Receiver Operating Characteristic Curves are Described by Kullback-Leibler Divergences, Entropy, 15(4), 1342-1356.

[8] Iverson, G. and Bamber, D. (1997), The generalized area theorem in signal detection theory, Choice, Decision and Measurement: Essays in Honor of R. Duncan Luce, editor A.A.J. Marley, Lawrence Erlbaum Associates, Inc., New Jersey.

[9] Johnson, D. H. and Dudgeon, D. E. 1993, Array Signal PROCessing: Concepts and Techniques, Prentice Hall, New Jersey.

[10] Killeen, P. R. and Taylor, T. J. (2004), Symmetric receiver operating characteristics, Journal of Mathematical Psychology, 48, 432-434.

[11] Krzanowski, W. J. and Hand, D. J. (2009), ROC Curves for Continuous Data, Chapman \& Hall/CRC, Boca Raton, FL.

[12] Lloyd, C. J. (2000), Regression models for convex ROC curves, Biometrics 56, 862-867.

[13] Marzban, C. (2004), The ROC curve and the area under it as performance measures, Weather and Forecasting, 19, 1106-1114.

[14] Mielniczuk, J. (1992), Grade estimation of Kullback-Leibler information number, Probability And Mathematical Statistics, 13, 139-147.

[15] Pepe, M. S. (2003), The Statistical Evaluation of Medical Tests for Classification and Prediction, OUP, Oxford.

[16] Taylor, M. M. (1967), Detectability theory and the interpretation of vigilance data, Acta Psychologica, 27, 390-399.

[17] Twengström, E., Sigvald, R., Svensson, C. and Yuen, J. (1998), Forecasting Sclerotinia stem rot in spring sown oilseed rape, Crop Protection, 17, 405-411. 
[18] Volkau, I., Prakash, B., Ananthasubramaniam A., Gupta, V., Aziz, A., Nowinski, W. L., (2006), Quantitative Analysis of Brain Asymmetry by Using the Divergence Measure: Normal-Pathological Brain Discrimination, Academic Radiology, 13(6), 752-758.

[19] Zhang, L., Tong, M. H., Marks, T. K., Shan, H., \& Cottrell, G. W. (2008), SUN: A Bayesian framework for saliency using natural statistics, Journal of Vision, 8(7):32, 1-20.

[20] Zhou, X. H., McClish, D. K. and Obuchowski, N. A. (2002), Statistical Methods in Diagnostic Medicine, Wiley, New York. 